\title{
New Feedback Functions for Synchronizing Chaotic Maps
}

\author{
M.K. ALI \\ Department of Physics, The University of Lethbridge, Lethbridge, Alberta T1K 3M4, Canada
}

(Received 20 August 1997)

\begin{abstract}
Synchronization of chaotic maps is studied using the method of variable feedback. A general method is presented for generating feedback functions for maps. These feedback functions are found very efficient. Our study shows that when the driver and the response systems are fed by common noise, the noise does not affect synchronization. With different but weak noise added to the driver and response systems, approximate synchronization persists.
\end{abstract}

Keywords: Chaos, Noise, Synchronization, Feedback, Maps, Control

PACS numbers: $05.45 .+\mathrm{b}, 05.40 .+\mathrm{j}$

Recently, synchronization of chaos has drawn increasing attention [1-4] from both foundational and applied considerations. One of the methods used for synchronization of chaos is known as the method of variable feedback [5]. It is a robust method but the feedback functions are not unique. Worse, there is no all encompassing general method available for finding working feedback functions. Synchronization of chaos in the presence of noise bears considerable practical importance. The main purpose of this work is twofold. First we present a general method that has worked well in finding efficient feedback functions in test cases of invertible and noninvertible maps. Second we apply the feedback functions to study synchronization of sample chaotic attractors with and without noise. As for synchronization of chaos with common noise, Maritan and Banavar [7] observed that two initially different trajectories of a chaotic attractor fed by common noise coalesce when calculations are carried out with finite precision. Lech et al. [8] and Pikovsky [9] have shown that if the calculations are done with higher precision the coalescence time $\tau$ (for maps $\tau=$ no. of iterations) increases exponentially and the procedure soon becomes practically impossible to implement. Clearly, the application of common noise for synchronization of chaotic attractors has inherent difficulties. In this work we will see that synchronization of chaotic attractors in the presence of common noise poses no additional difficulty [2]. 


\section{THE FEEDBACK FUNCTIONS}

The method for generating feedback functions is in order here. Suppose, we have to synchronize the map

$X_{n+1}(i)=f_{i}\left(\mathbf{X}_{n}\right)=f_{i}\left(X_{n}(1), X_{n}(2), \ldots, X_{n}(L)\right)$

with its replica

$$
Y_{n+1}(i)=f_{i}\left(\mathbf{Y}_{n}\right)=f_{i}\left(Y_{n}(1), Y_{n}(2), \ldots, Y_{n}(L)\right)
$$

where $n$ and $i$ are discrete time and space variables, $L$ is the total number of space variables and $f_{i}$ is a function of its arguments. The method of variable feedback involves defining feedback functions $G_{i}\left(\mathbf{X}_{n}, \mathbf{Y}_{n}\right)$ and iterating Eqs. (1) and (3) simultaneously $[3,5]$ :

$Y_{n+1}(i)=f_{i}\left(Y_{n}(1), Y_{n}(2), \ldots, Y_{n}(L)\right)+G_{i}\left(\mathbf{X}_{n}, \mathbf{Y}_{n}\right)$.

The starting state vectors $\mathbf{X}_{0}=\left[X_{0}(1), X_{0}(2), \ldots\right.$, $\left.X_{0}(L)\right]^{\mathrm{T}}$ and $\mathbf{Y}_{0}=\left[Y_{0}(1), Y_{0}(2), \ldots, Y_{0}(L)\right]^{\mathrm{T}}$ are different. The superscript $\mathrm{T}$ means transpose of the row vectors. Synchronization is said to have been achieved if the state vectors satisfy the condition $\mathbf{X}_{m} \rightarrow \mathbf{Y}_{m}$ as $m \rightarrow \infty$. After synchronization has been achieved, the feedback functions $G_{i}\left(\mathbf{X}_{n}, \mathbf{Y}_{n}\right)$ vanish for all $i$. The system described by Eq. (1) is called the driver system while that described by Eq. (3) is called the response system. Pyragas [5] has used

$$
G_{i}\left(\mathbf{X}_{n}, \mathbf{Y}_{n}\right)=-k\left[Y_{n}(i)-X_{n}(i)\right]
$$

where $k$ is a suitably chosen constant. Peng et al. [6] have used

$$
G_{i}\left(\mathbf{X}_{n}, \mathbf{Y}_{n}\right)=-\sum_{j} k_{i j}\left[Y_{n}(j)-X_{n}(j)\right]
$$

where $k_{i j}$ are suitably chosen constants. Equations (4) and (5) provide linear feedback functions. However, the $G_{i}\left(\mathbf{X}_{n}, \mathbf{Y}_{n}\right)$ do not have to be restricted to linear forms [3] for synchronizing chaotic and hyperchaotic systems. We now derive our main result. Define, using Eqs. (1) and (2), $\Delta Y_{n+1}(i)=Y_{n+1}(i)-X_{n+1}(i)=f_{i}\left(\mathbf{Y}_{n}\right)-f_{i}\left(\mathbf{X}_{n}\right)$.
There is a $\beta$ for which the feedback functions given by

$$
G_{i}\left(\mathbf{X}_{n}, \mathbf{Y}_{n}\right)=-\beta \Delta Y_{n+1}(i), \quad i=1,2, \ldots, L
$$

are found to synchronize the chaotic maps. Comparing Eqs. (4)-(6), we notice that Eq. (6) contains $Y_{n+1}(i)$ and $X_{n+1}(i)$ while Eqs. (4) and (5) involve $Y_{n}(i)$ and $X_{n}(i)$. This difference in Eqs. (4)-(6) gives us new feedback functions. First of all, we notice that when $\beta=1$, Eqs. (1) and (3) become identical and we have a trivial case $\mathbf{Y}=\mathbf{X}$ in one time step. Away from this trivial case, there is an infinite set of values of $\beta$ in the interval $\beta_{\min } \leq \beta<1$ for which the above feedback function of Eq. (6) is found to synchronize the two identical chaotic maps. With $\beta=1$, nontrivial feedback functions can be found by using an approximation of $\Delta Y_{n+1}(i)$. To the first order approximation, we have

$$
\begin{aligned}
& \Delta Y_{n+1}(i) \\
& \quad=\sum_{j=1}^{L} \frac{\partial f_{i}\left(X_{n}(1), X_{n}(2), \ldots, X_{n}(L)\right)}{\partial X_{n}(j)} \Delta Y_{n}(j) .
\end{aligned}
$$

This approximation for the $\Delta Y_{n+1}(i)$ gives the new feedback functions as

$$
\begin{aligned}
& G_{i}\left(\mathbf{X}_{n}, \mathbf{Y}_{n}\right)=-\beta \\
& \quad \times \sum_{j=1}^{L} \frac{\partial f_{i}\left(X_{n}(1), X_{n}(2), \ldots, X_{n}(L)\right)}{\partial X_{n}(j)} \Delta Y_{n}(j) .
\end{aligned}
$$

Comparing Eqs. (4), (5) and (8), we notice that the coefficients of $\Delta Y_{n}(m)$, where $\Delta Y_{n}(m)=$ $Y_{n}(m)-X_{n}(m)$, in Eq. (8) are functions of $\mathbf{Y}_{n}$ and $\mathbf{X}_{n}$ while they are constants in Eqs. (4) and (5). These new feedback functions are the main results of this note. When Eq. (8) is used in Eq. (3) and the dynamics proceeds with $\beta=1$, it may happen that at some time step $\mu$ the $G_{i}\left(\mathbf{X}_{n}, \mathbf{Y}_{n}\right)$ yield the response state vector $\mathbf{Y}_{\mu}$ outside the basin of attraction. If it happens so, there are at least two ways to avoid this situation: (1) do not use the feedback at this time step and let iterations continue till the feedback term gives a point in the basin of attraction; or (2) set $\beta$ to a value in 
the interval $0 \leq \beta<1$ so that $\mathbf{Y}_{\mu}$ is in the basin of attraction. The step next to $\mu$ is started with $\beta=1$ and the procedure is repeated. The above feedback functions of Eq. (8) are linear in $\Delta \mathbf{Y}_{n}$ and are generalizations of the schemes of Pyragas [5] and Peng et al. [6]. These generalized feedback functions widen the areas of application of the feedback approach. It is interesting to note that feedback functions obtained by the general form of Eq. (8) synchronize identical chaotic maps. Higher orders in $\Delta \mathbf{Y}_{n}$ may be tried. However, the above simple but general procedure have worked very well in all of our test cases. We now illustrate applications of these new feedback functions with the help of sample chaotic maps.

\section{SYNCHRONIZATION OF SAMPLE CHAOTIC ATTRACTORS}

Using feedback functions obtained from the scheme of Eq. (8) in Eq. (3), we consider synchronization of three discrete time chaotic maps.

\section{A. The logistic map}

$$
\begin{gathered}
X_{n+1}=4 X_{n}\left(1-X_{n}\right), \\
Y_{n+1}=4 Y_{n}\left(1-Y_{n}\right)+\beta 4\left(Y_{n}-X_{n}\right)\left(2 X_{n}-1\right) .
\end{gathered}
$$

\section{B. The Ikeda map}

$$
X_{n+1}(1)=1+c_{2}\left[X_{n}(1) \cos \left(\tau_{x}\right)-X_{n}(2) \sin \left(\tau_{x}\right)\right],
$$$$
X_{n+1}(2)=c_{2}\left[X_{n}(1) \sin \left(\tau_{x}\right)+X_{n}(2) \cos \left(\tau_{x}\right)\right],
$$

$$
\begin{aligned}
Y_{n+1}(1)= & 1+c_{2}\left[Y_{n}(1) \cos \left(\tau_{y}\right)\right. \\
& \left.-Y_{n}(2) \sin \left(\tau_{y}\right)\right]+G_{1}, \\
Y_{n+1}(2)= & c_{2}\left[Y_{n}(1) \sin \left(\tau_{y}\right)+Y_{n}(2) \cos \left(\tau_{y}\right)\right]+G_{2},
\end{aligned}
$$

$$
\begin{aligned}
G_{1}= & -\beta c_{2}\left[\left(\cos \left(\tau_{x}\right)-g_{1} t_{1}\right)\left(Y_{n}(1)-X_{n}(1)\right)\right. \\
& \left.-\left(\sin \left(\tau_{x}\right)+g_{1} t_{2}\right)\left(Y_{n}(2)-X_{n}(2)\right)\right], \quad(15) \\
G_{2}= & -\beta c_{2}\left[\left(\sin \left(\tau_{x}\right)+g_{2} t_{1}\right)\left(Y_{n}(1)-X_{n}(1)\right)\right. \\
& \left.+\left(\cos \left(\tau_{x}\right)+g_{2} t_{2}\right)\left(Y_{n}(2)-X_{n}(2)\right)\right],(16)
\end{aligned}
$$

$$
\begin{gathered}
t_{1}=\frac{2 X_{n}(1)\left(c_{1}-\tau_{x}\right)^{2}}{c_{3}}, \quad t_{2}=\frac{2 X_{n}(2)\left(c_{1}-\tau_{x}\right)^{2}}{c_{3}}, \\
\tau_{x}=c_{1}-\frac{c_{3}}{1+X_{n}(1)^{2}+X_{n}(2)^{2}}, \\
\tau_{y}=c_{1}-\frac{c_{3}}{1+Y_{n}(1)^{2}+Y_{n}(2)^{2}}, \\
g_{1}=X_{n}(1) \sin \left(\tau_{x}\right)+X_{n}(2) \cos \left(\tau_{x}\right), \\
g_{2}=X_{n}(1) \cos \left(\tau_{x}\right)-X_{n}(2) \sin \left(\tau_{x}\right), \\
c_{1}=0.4, \quad c_{2}=0.9, \quad c_{3}=6 .
\end{gathered}
$$

\section{The Henon map}

$$
\begin{aligned}
& X_{n+1}(1)= 2.12-X_{n}(1)^{2}-0.3 X_{n}(2), \\
& X_{n+1}(2)=X_{n}(1), \\
& Y_{n+1}(1)= 2.12-Y_{n}(1)^{2}-0.3 Y_{n}(2) \\
&+\beta\left[2 X _ { n } ( 1 ) \left(Y_{n}(1)-X_{n}(1)\right.\right. \\
&\left.+0.3\left(Y_{n}(2)-X_{n}(2)\right)\right], \\
& Y_{n+1}(2)=Y_{n}(1)+\beta\left[X_{n}(1)-Y_{n}(1)\right] .
\end{aligned}
$$

The coefficients of the Ikeda and Henon maps are taken from [10]. Starting with different random state vectors $\mathbf{X}_{\mathbf{0}} \neq \mathbf{Y}_{\mathbf{0}}$, we have iterated the three maps. Table I shows the efficiency of the feedback functions in synchronizing these sample cases. The logistic, Ikeda and Henon maps synchronize with their replica after 4,8 and 5 iterations respectively. This fast rate of convergence to perfect synchronization of chaotic trajectories can be quite useful. It has, for example, an advantage over methods with slow rate of convergence in studying synchronization in the presence of noise. Employing an efficient method to synchronize dynamical chaos, one is in a better situation to deal with the noise. Our numerical results show that the synchronization times are not affected when common noise is added to the driver and response systems. This means that synchronization is always achieved in the presence of common noise with the same efficiency as in the absence of noise. If the driver or the response 
system is subjected to noise or if both the driver and the response systems are fed by different noise terms, then we have three different scenarios: (1) if the noise is added sporadically at a time interval larger than the synchronization time then the systems are desynchronized and synchronized at the frequency of addition of noise; (2) if weak noise terms are added at every iteration, then approximate synchronization is maintained; (3) if strong noise is added at every time step, no synchronization is possible. These three scenarios are illustrated in Figs. 1-3 for the Henon map. Similar results are obtained with the other two maps of our study. In these figures, desynchronization is measured by deviation from 0 of the function $\Delta(n)$ where

$$
\Delta(n)=\sqrt{\sum_{i=1}^{L}\left(Y_{n}(i)-X_{n}(i)\right)^{2}} .
$$

For feeding noise, four different random numbers $W \eta_{i}, i=1,2,3,4$ with each $\eta_{i}$ lying in the interval $(-1,2)$ were added to $X_{n}(1), X_{n}(2), Y_{n}(1)$ and $Y_{n}(2)$. Here $W$ denotes the strength of the noise. Only those $\eta$ 's were used for which the noise terms $\left(W \eta_{i}\right)$ did not drive the state vectors outside the basins of attraction. For Figs. 1, 2 and 3, we used $W=1, W=0.1$ and $W=1$ with the noise added at the time intervals 10,1 and 1 respectively. As expected, Fig. 1 shows periodic desynchronization

TABLE I Synchronization of the Logistic map (Eqs. (9) and (10)), Ikeda map (Eqs. (11)-(20)) and Henon map (Eqs. (21)-(25)) in the absence of noise. The initial states are chosen from a random number generator. The table shows that synchronization occurs after $n=4,8$ and 5 iterations in these maps respectively. Although the synchronized numbers quoted in the table are shown to agree to nine decimal places, they agreed to all figures in our double precision calculations. Some initial conditions required less and some required more than the quoted number of iterations to synchronize but in all the test cases synchronization were achieved within about 10 iterations

\begin{tabular}{|c|c|c|c|c|c|c|}
\hline \multirow[t]{2}{*}{$\mathbf{X}_{n}, \mathbf{Y}_{n}$} & \multicolumn{2}{|c|}{ Logistic map } & \multicolumn{2}{|c|}{ Ikeda map } & \multicolumn{2}{|c|}{ Henon map } \\
\hline & $n=0$ & $n=4$ & $n=0$ & $n=8$ & $n=0$ & $n=5$ \\
\hline$X_{n}(1)$ & 0.89362 & 0.678047409 & 0.81680 & 1.230491899 & 1.04600 & -0.608373053 \\
\hline$Y_{n}(1)$ & 0.29947 & 0.678047409 & 0.00565 & 1.230491899 & -0.36625 & -0.608373053 \\
\hline$X_{n}(2)$ & & & 0.15381 & -1.152648407 & 1.23846 & 1.618741161 \\
\hline$Y_{n}(2)$ & & & 0.13486 & -1.152648407 & 1.16939 & 1.618741161 \\
\hline
\end{tabular}

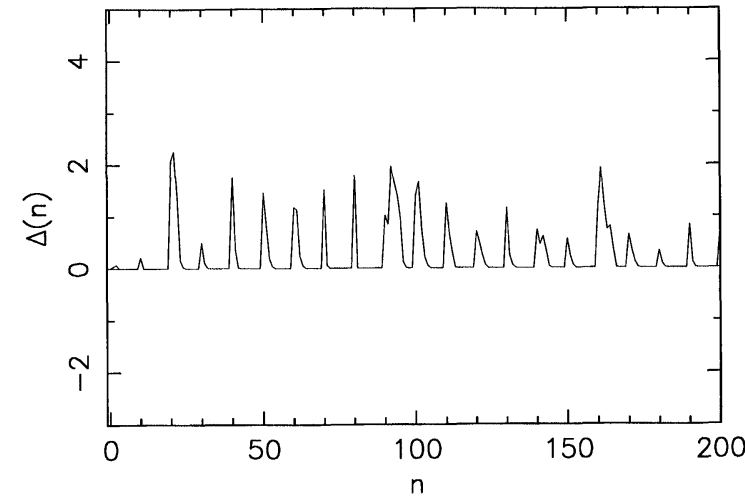

FIGURE 1 Synchronization of the Henon map in the presence of noise with $W=1$. The noise in the form of random numbers are added to $X_{n}(1), X_{n}(2), Y_{n}(1)$ and $Y_{n}(2)$ at a frequency of 10 discrete time steps. The desynchronization peaks are seen at a regular time interval of 10 steps.

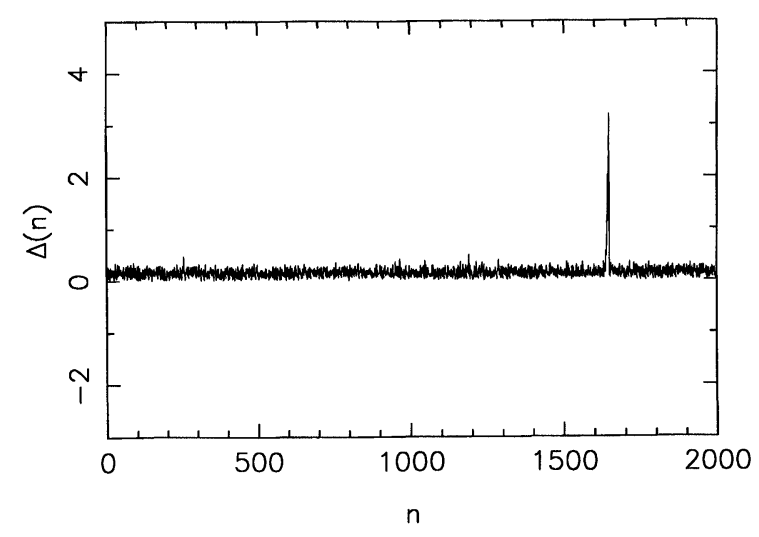

FIGURE 2 Synchronization of the Henon map in the presence of noise with $W=0.1$. The noise is added to $X_{n}(1)$, $X_{n}(2), Y_{n}(1)$ and $Y_{n}(2)$ at every discrete time step. 


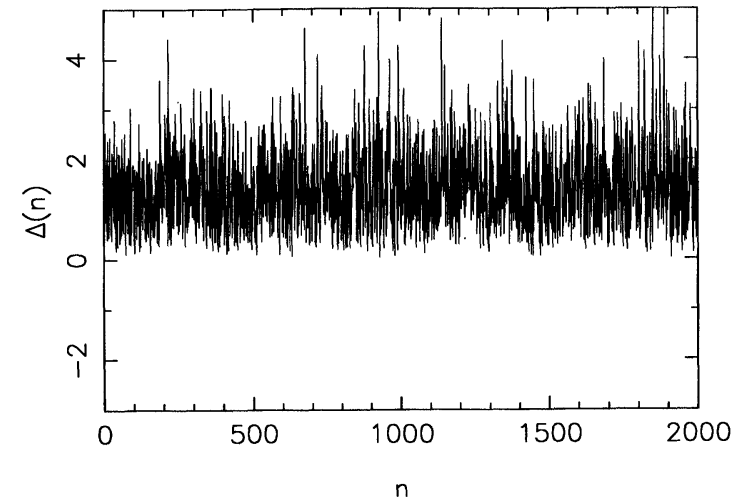

FIGURE 3 Same as Fig. 2 with $W=1$.

and synchronization at the frequency of 10. Fig. 2 shows approximate synchronization in the presence of relatively weak noise. This behavior simulates some experimental situation and it has become possible here with our fast and accurate method for synchronization of dynamical chaos. By controlling $W$, we can achieve different degrees of synchronization. It is striking that the two chaotic systems stay close to each other in the presence of noise even after big random desynchronization bursts. It may be hard to see this behavior when a synchronization method with slow rate of synchronization is used. Figure 3 shows that with strong noise added at every time step, no synchronization is possible. It may be possible, under certain conditions, to find feedback functions with faster synchronization rate. The merit of the scheme presented here is that it is general and it does not require any guessing about the nature of the feedback functions. On the basis of our numerical results of the three cases, we conjecture that our findings are true in general.

\section{Acknowledgement}

This research has been supported by an NSERCC grant.

\section{References}

[1] L.M. Pecora and T.L. Carroll, Phys. Rev. Lett., 64, 821 (1990); E. Ott, C. Grebogi and J.A. Yorke, Phys. Rev. Lett., 64, 1196 (1990); Y.C. Lai and C. Grebogi, Phys. Rev. E, 47, 2357 (1993).

[2] M.K. Ali, Phys. Rev. E, 55, 4804 (1997).

[3] M.K. Ali and Jin-Qing Fang, Phys. Rev. E, 55, 5285 (1997).

[4] M.K. Ali and Jin-Qing Fang, Discrete Dynamics in Nature and Society (to appear in volume 3), 1997.

[5] K. Pyragas, Phys. Lett. A, 170, 421 (1992); Y. Nagai and Y. Lai, Phys. Rev. E, 51, 3842 (1995); H.D.I. Abarbanel, N.F. Rulkov and M.M. Sushchik, Phys. Rev. E, 52, 214 (1995); G. Hu, Z. Qu and K. He, Int. J. Bif. and Chaos, 5, 901 (1995); He Kaifen and Hu Gang, Phys. Rev. E, 53, 2271 (1996); and references therein.

[6] J.H. Peng, E.J. Ding, M. Ding and W. Yang, Phys. Rev. Lett., 76, 904 (1996).

[7] A. Maritan and J.R. Banavar, Phys. Rev. Lett., 72, 1451 (1994).

[8] Lech Longa, Evaldo M.F. Curado and Fernando A. Oliveira, Phys. Rev. E, 54, R2201 (1996).

[9] A.S. Pikovsky, Phys. Rev. Lett., 73, 2931 (1994).

[10] J.A. Yorke, "Dynamics" (University of Maryland, College Park, Maryland, USA, 1991). 


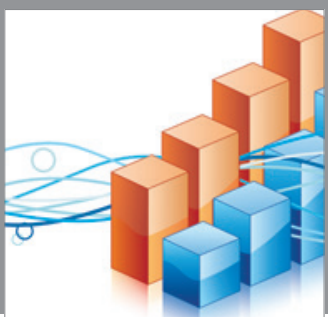

Advances in

Operations Research

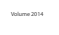

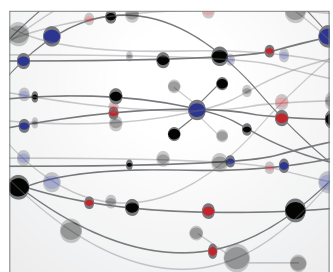

\section{The Scientific} World Journal
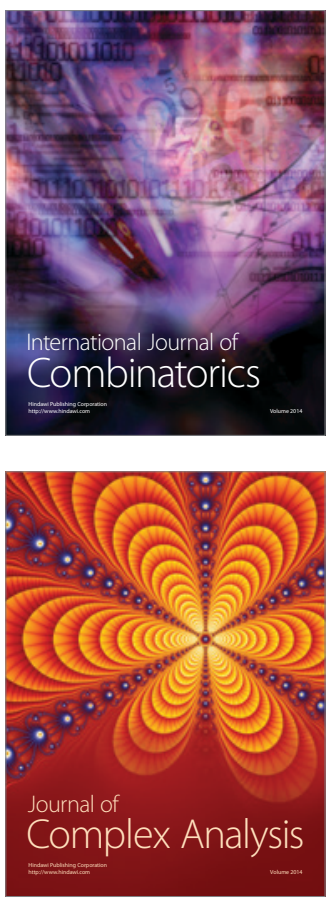

International Journal of

Mathematics and

Mathematical

Sciences
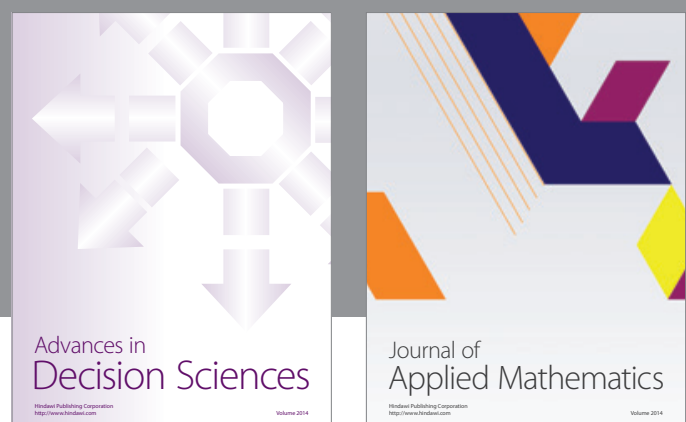

Journal of

Applied Mathematics
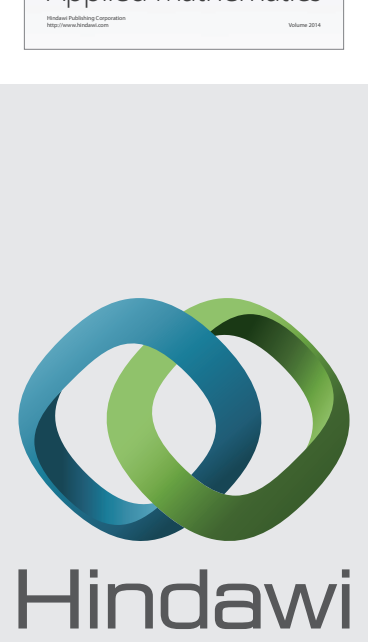

Submit your manuscripts at http://www.hindawi.com
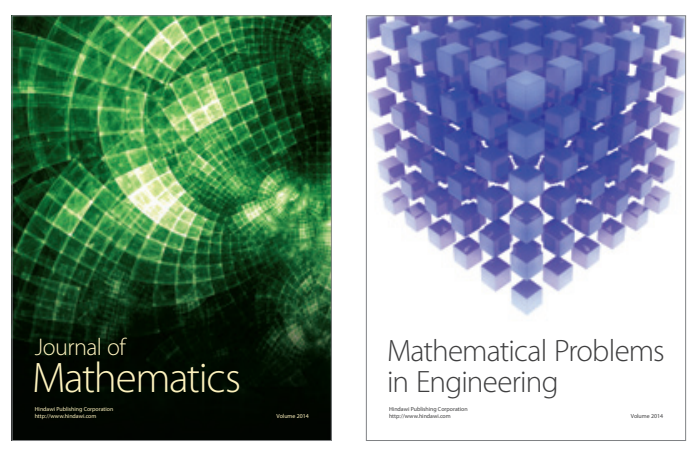

Mathematical Problems in Engineering
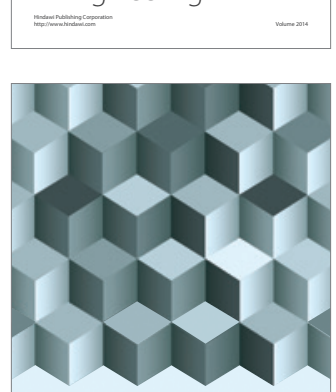

Journal of

Function Spaces
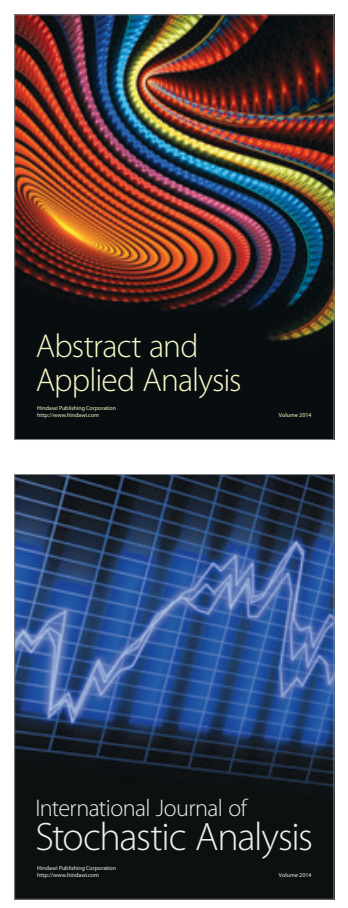

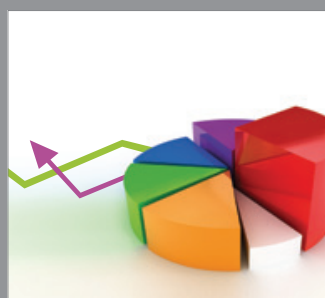

ournal of

Probability and Statistics

Promensencen
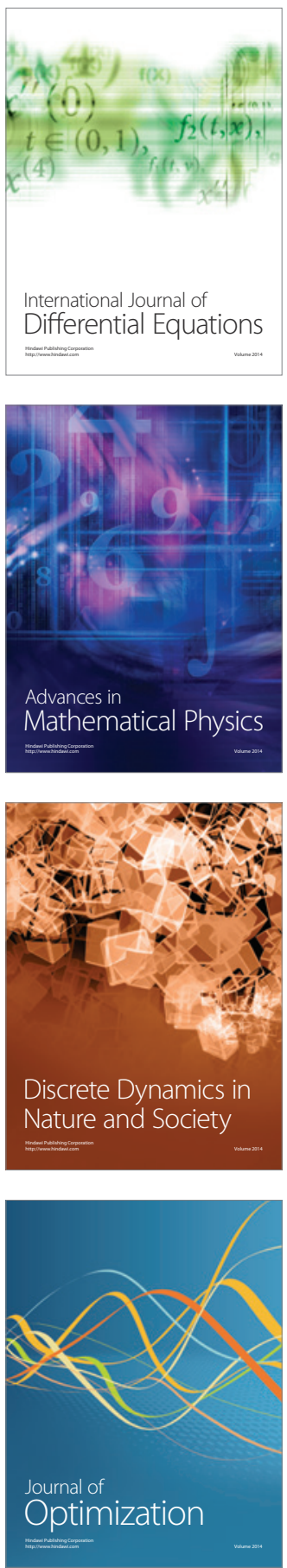